\title{
The Use of the Grey Level SAT to Find the Salient Cavities in Echocardiograms
}

\author{
David R Bailes \\ Wolfson Image Analysis Unit \\ Department of Medical Biophysics \\ University of Manchester \\ Oxford Road \\ Manchester M13 9PT
}

The aim of this work is to find out whether the grey level symmetric axis transform ( SAT) is a useful grey level shape description for finding the salient cavities in echocardiograms. The centres of the maximal discs of the grey level SAT are calculated using mathematical morphology as described by Peleg and Rosenfeld, and these centres are grouped to form symmetry surfaces. These surfaces are described as a graph in which each vertex has associated with it a description of a path from the top to the bottom of a symmetry surface, and two vertices are connected if their two paths are adjacent. The subgraphs of this graph which correspond to the salient cavities in the image are found by growing subgraphs from seed vertices. Finally, the boundaries of the cavities are reconstructed from these subgraphs. Our initial results are promising, although further work is needed to make the method more robust.

The work described in this paper was done as part of the cardiac subgroup of the Alvey project MMI-134: Model Based Processing of Radiological Images. The aim of this subgroup is to automatically locate the boundaries of the left ventricle in time sequences of images generated by both ultrasound and MRI, although in this paper we only consider ultrasound images. The motivation for this is that the performance of the left ventricle can be quantified from these boundaries, but that tracing the boundaries manually is time consuming. A review of quantitation in echocardiography is given by Skorton and Collins [1]. An example of an echocardiogram showing an apical four chamber view of the heart is shown in Fig 1.

Nearly all the existing computer aided methods for finding the boundaries of the left ventricle rely on an operator pointing to the centre of the left ventricle or drawing its boundary in at least one of the time frames. We have decided to do this automatically by finding the main features in the image, and then matching these to a model.

The cavities are amongst the main features in the images, and some these correspond to the heart chambers. The grey level symmetric axis transform ( SAT ) would seem to be a useful grey level shape description for finding the salient cavities in the image since it makes the depth of the cavities explicit. Many of

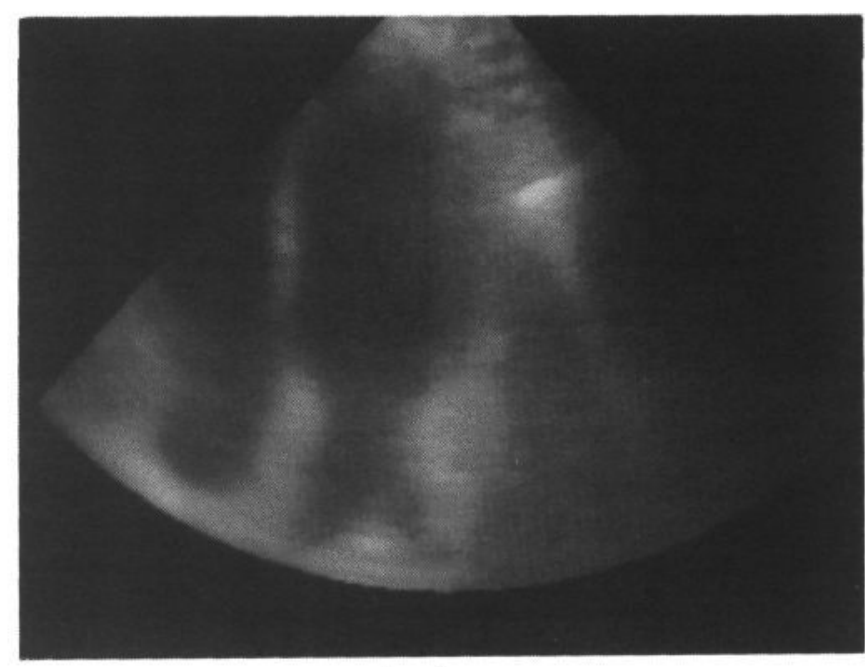

( a )

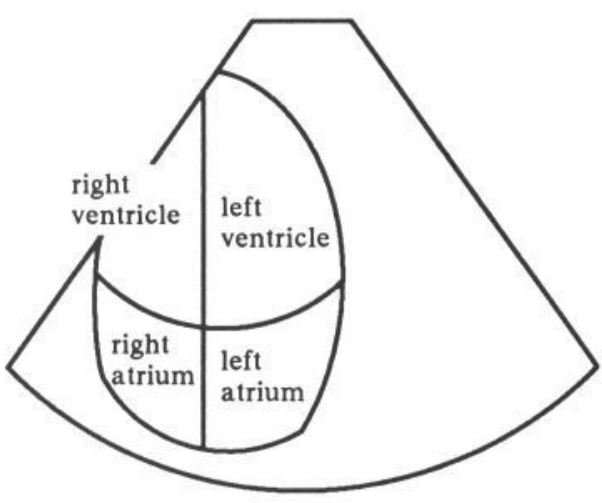

( b )

Figure 1. An example of a four chamber apical view which has been averaged over a heart cycle. (a) $256 \times 256$ image, (b) schematic diagram.

the methods which have been described for using the SAT to segment images have segmented the SAT at its branch points [ 2,3,4]. However this often results in subparts which are not particularly intuitive, and we do not use this procedure.

\section{THE GREY LEVEL SAT}

A number of ways of generalising the binary SAT to grey level images have been suggested, and we have used the

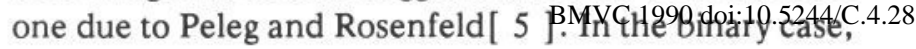


( a )

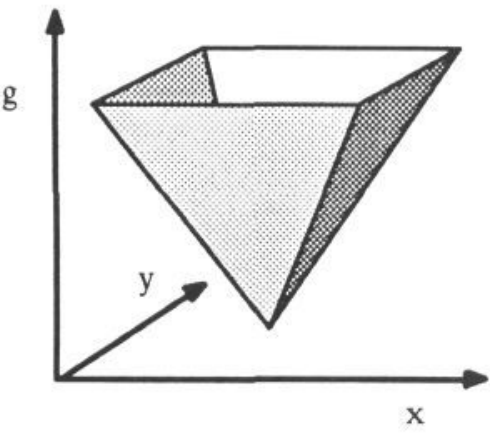

( b )

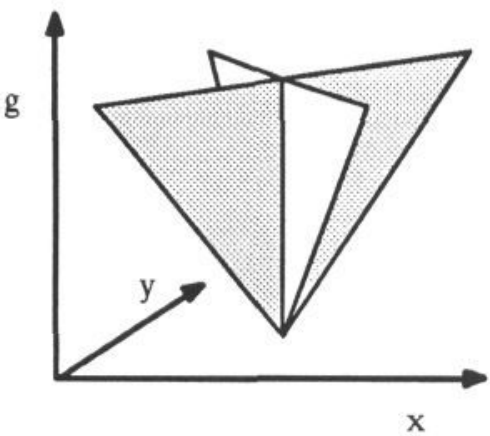

(c)

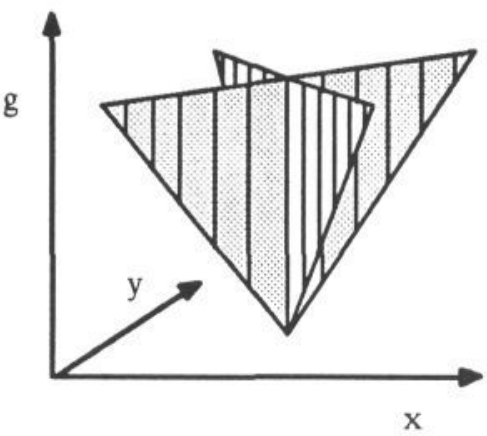

(d)

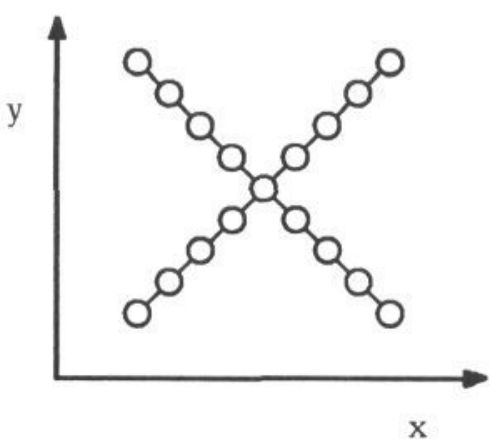

Figure 2. An example of a grey level SAT. (a) a grey level cavity which has the shape of a square pyramid, (b) the symmetry surfaces of the cavity, (c) the surface paths on the symmetry surfaces, (d) the gsat graph the SAT of either the image or the complement of the image can be calculated. Similarly in the grey level case, one can calculate the grey level SAT of either the three dimensional region below the surface defined by the grey level function, or the region above the surface. The grey level SAT of the region above/below the surface is the locus of the centres of the maximal discs whose plane is perpendicular to the grey level axis, and which are included in the region above/below the surface, together with their radii. In the remainder of this paper we consider the description of the region above the surface, and refer to it as simply the grey level SAT.

The locus of the centres of the maximal discs forms a number of symmetry surfaces. Figs $2 a$ and $2 b$ show a grey level cavity which has the shape of a square pyramid, and the the symmetry surfaces of its grey level SAT. There are a number of ways in which these surfaces could be described, and we have chosen to describe them as an undirected graph, which we call a gsat graph. Each vertex has associated with it a description of a path from the top to the bottom of a symmetry surface, and we refer to the these paths as surface paths. Two vertices are connected if their surface paths are adjacent. The surface paths and the gsat graph of the grey level SAT in Fig $2 \mathrm{~b}$ are shown in Figs $2 \mathrm{c}$ and $2 \mathrm{~d}$ respectively. For each surface path we calculate the following parameters which are used for finding the cavities:

a) average spatial position

b) average radius of the maximal discs

c) difference in grey level between the top and the bottom of the path

d) grey level of the bottom of the path

In the diagrams of gsat graphs in this paper, the position of each vertex is the average spatial position of the associated surface path.

In general each surface path takes the route of steepest descent from the top to the bottom of the symmetry surface so that the path, and hence its description of the surface, is as spatially localised as possible. However this causes the gsat graph to be unnecessarily complicated at the intersections of surfaces if the intersection is not parallel to the grey level axis. An example of such an intersection together with its gsat graph is shown in Figs $3 \mathrm{a}$ and $3 \mathrm{~b}$. A solution is to relax the steepest descent requirement at intersections and in their immediate vicinity. The modified surface paths and gsat graph are shown in Figs $3 \mathrm{c}$ and 3d.

It is also desirable to segment some of the surface paths. This is most easily seen by considering the grey level SAT of a one dimensional grey level function. Fig $4 \mathrm{a}$ shows a function which is a valley which is made up of two parts which have different widths, together with the grey level SAT which consists of one axis. (An axis in the one dimensional case corresponds to a surface path in the two dimensional case.) It is useful for each of the two parts of the valley to have its own axis, and so the axis has to be segmented. The function $\mathrm{dg} / \mathrm{dr}$ against $\mathrm{r}$ for the axis is shown in Fig $4 b$, where $r$ is the radius of 

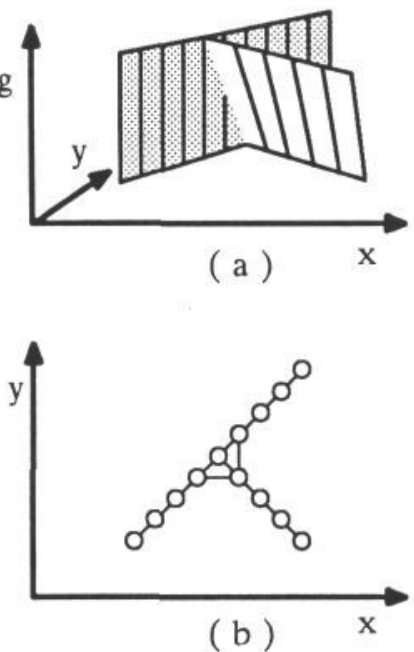

Figure 3. Surface paths at the intersections of surfaces. If the surface paths take the route of steepest descent as in (a), then the resulting gsat graph (b) is unnecessarily complicated. If the steepest descent requirement is relaxed in the vicinity of the intersection (c), then the gsat graph (d) is simplified.
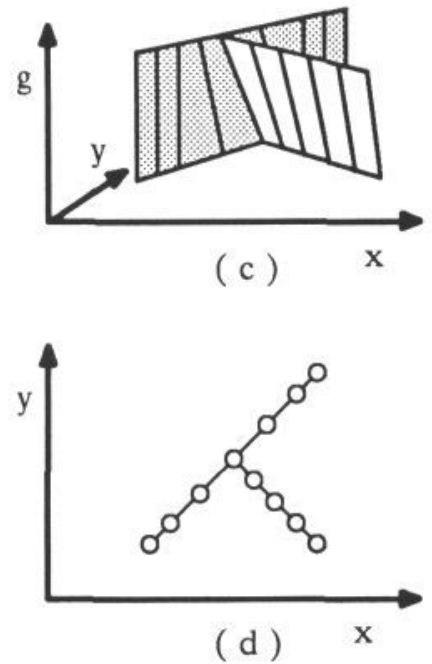
,

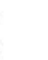




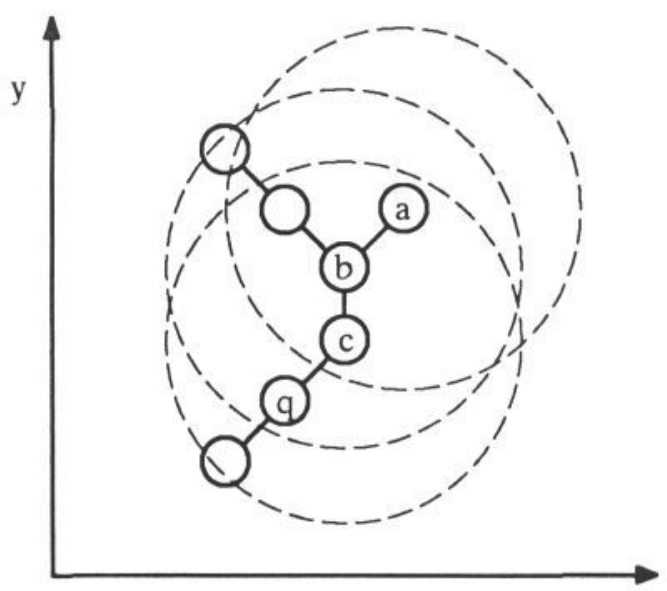

(a)

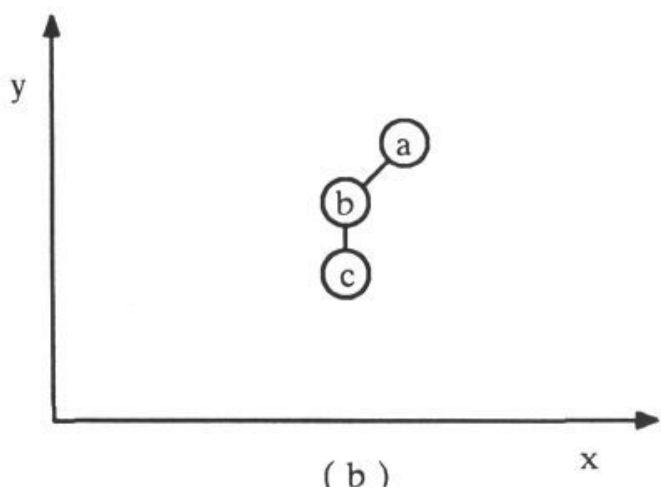

Figure 5. Growing a subgraph. (a) A gsat graph. For each of the vertices $a, b$ and $c$, a dashed circle is shown which has the average radius of the vertex. (b) The subgraph which is being grown.

e) The vertices in the subset graphs are linked together to form surface paths which each go from the top to the bottom of a symmetry surface. These paths take the route of steepest descent, except at the intersections of surfaces, as discussed in the previous section.

f) Some of the surface paths are segmented.

g) The gsat graph is constructed from these paths. Two gsat vertices are connected if any subset vertex in one path is connected in a subset graph to a subset vertex in the other path.

h) For each path we calculate the following parameters which will be used for the segmentation described in the next section: the average position; the average radius; the difference in grey levels between the top and the bottom of the path; the grey level of the bottom of the path.

\section{FINDING THE BOUNDARIES OF THE SALIENT CAVITIES}

The boundaries of the salient cavities of the image are obtained by finding the subgraphs of the gsat graph which correspond to these cavities, and then reconstructing the boundaries from these subgraphs.

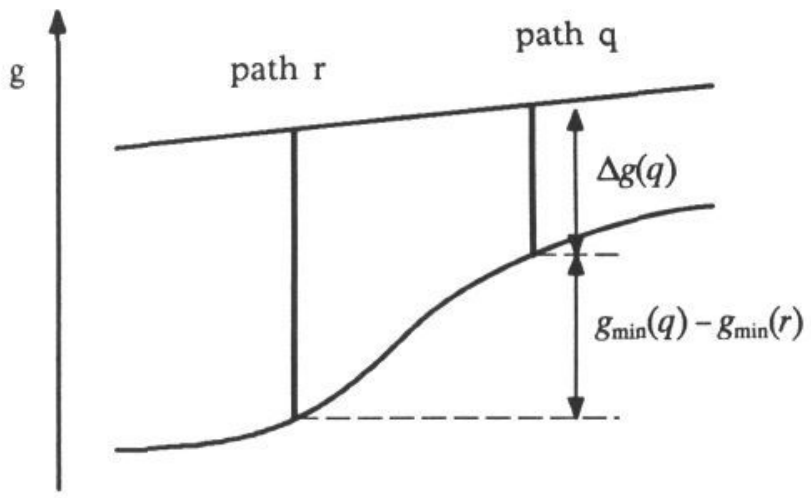

Figure 6. The compatibility of two vertices

Our method has the following desirable behaviour when an image is scaled:

a) If the grey levels of the image are scaled, then the shape and size of the cavities remain constant.

b) If the image is spatially scaled, then the shapes of the cavities remain constant, and their sizes are scaled.

The method for finding the subgraphs of the gsat graph which correspond to the salient cavities consists of two parts: select a seed vertex; grow a subgraph from this vertex. This is repeated to find as many cavities as are required.

The seed vertex is selected by finding the vertex in the gsat graph which has not already been used for a subgraph, and which has the highest value of the difference in grey level between the top and the bottom of the surface path.

At the edges of a cavity, the grey levels of the bottoms of the symmetry surfaces increase, and it is this increase in grey level which we use to determine when to stop growing the subgraph. More precisely, the subgraph initially consists of the seed vertex, and a vertex is added to the subgraph if the following conditions are met:

a) it has not already been used for a subgraph.

b) it is connected in the main graph to a vertex which is already part of the subgraph. We will refer to this vertex as vertex c.

c) it is compatible with the subgraph.

Condition b) is illustrated in Fig 5, where the vertex $q$ is connected to vertex c, which is already part of the subgraph being grown.

The compatibility of a vertex $\mathrm{q}$ with a subgraph is defined in two parts as follows. Firstly vertex $q$ is compatible with the vertex $r$ if:

$$
\frac{g_{\min }(q)-g_{\min }(r)}{\Delta g(q)}<\text { threshold }
$$

where $g_{\min }$ is the grey level of the bottom of a surface path, and $\Delta g$ is the difference in grey level between the top and the bottom of a surface path ( see Fig 6 ).

Secondly, let $\mathrm{T}$ be the set of vertices such that each vertex is a member of the subgraph, and the distance between it and vertex $q$ is less than the average radius of 


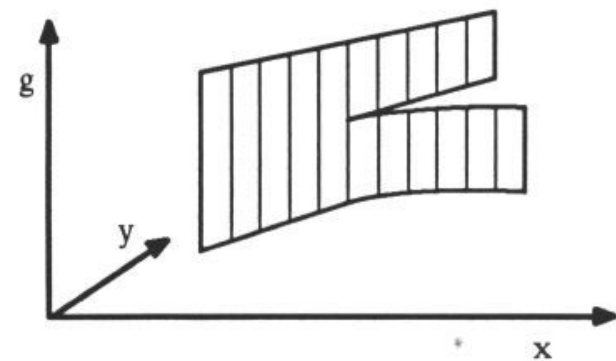

Figure 7. A symmetry surface which divides into an upper and a lower surface.

the vertex. In Fig 5 the subgraph being grown contains the vertices $a, b$ and $c$ and for each of these vertices a circle is shown which has the average radius of the vertex. The set $T$ consists of the vertices $b$ and $c$, since the vertex $q$ lies within the radii of the vertices $b$ and $c$, but not a.

Combining the two parts, vertex $q$ is compatible with the subgraph if it is compatible with all the vertices in the set $\mathrm{T}$. The first part of the definition of compatibility involves a threshold. As this threshold is increased the subgraphs in general gradually expand, and at certain values pairs of subgraphs merge. It may be necessary to run the method at a number of values of the threshold, and for some higher level reasoning to decide which threshold produces the most meaningful cavities. We currently use a threshold of about 0.4 .

If a vertex $q$ fulfils the conditions a) and b) above, but is not compatible with the subgraph, then that vertex and some of the neighbouring vertices are labelled so that they are not used for future subgraphs. This is done to prevent subsequent subgraphs from getting too close to previous subgraphs, and to stop vertices at the edge of this cavity being chosen as seed vertices. The labelled vertices are those that lie within the average radius of vertex c ( defined in condition b) ), and which can be reached from vertex $\mathrm{q}$ by $\mathrm{a}$ path which has monotonically increasing values of the grey level of the bottom of the surface path.

A complication arises at places where a symmetry surfaces surface divides into an upper and a lower surface. An example of this is shown in Fig 7. The upper and lower surfaces describe two different cavities, the lower one lying inside the upper one. The implications for growing subgraphs are firstly that a subgraph cannot contain both vertices on the upper surface and vertices on the lower surface. Secondly, if the subgraph has grown along the main surface and has reached the path where the surface divides, the compatibility rule defined above may well prevent the surface from growing any further. We do not think that it is possible to decide at this low level whether or not the surface should continue to grow, and so we generate three options, and leave the decision to some higher level reasoning. The three options are: a) Use the definition of compatibility defined above.

b) Grow along the lower surface, but restrict the set of vertices $\mathrm{T}$ to being on the lower surface.

c) Grow along the upper surface, but restrict the set of vertices $\mathrm{T}$ to being on the upper surface.

From each subgraph we reconstruct the boundary of the cavity as follows. For each vertex on the subgraph a filled circle is drawn which has the average radius of the vertex, and a centre at the average position of the vertex. Then the boundary of the cavity is the boundary of the region formed by the union of these circles.

\section{RESULTS}

We have applied the above method to only a small number of echocardiograms, and so it is not possible to give a reliable assessment of its performance. The grey level SAT of the image in Fig 1a is shown in Fig 8, where (a) shows the gsat graph superimposed on the original image, the position of each vertex being the average spatial position of the associated surface path, and (b), (c) and (d) show the values of the other parameters which describe the surface paths. The boundaries of the 5 most salient cavities which were found by the method described above are shown in Fig 9. It can be seen that by reference to Fig $1 \mathrm{~b}$ that 4 of the 5 cavities correspond to cardiac chambers.

The method is implemented in a mixture of Pascal and $\mathrm{C}$, and takes about one minute on a Sun $3 / 160$.

\section{CONCLUSIONS}

Our initial results have shown that the grey level SAT is a useful image description for finding the salient cavities in echocardiograms, but that further work is needed to make the method more robust. We have found that, unlike the binary case, ensuring the spatial connectivity of the grey level SAT is not the major problem, but that segmenting the surface paths is an area of greater difficulty, and needs further work.

\section{ACKNOWLEDGEMENTS}

Andrew Hill for the software for the subsets graphs, and for display software. Dr D. C. Beeton of Wythenshawe Hospital for supplying the echocardiograms. This work was funded by SERC as part of IED/ALVEY project MMI-134.

\section{REFERENCES}

1. Skorton, D.J. and Collins, S.M. “Quantitation in echocardiography", Cardiovasc. Intervent. Radiol., vol. 10 ( 1987 ), pp 316-331.

2. Blum, H. and Nagel, R.N. "Shape description using weighted symmetric axis features", Pattern Recognition, vol. 10 ( 1978 ), pp 167-180.

3. Nackman, L.R., and Pizer, S.M. "Three-dimensional shape description using the symmetric axis transform 1: theory", IEEE Trans. PAMI, vol. 7 ( 1985 ), pp 187-202. 


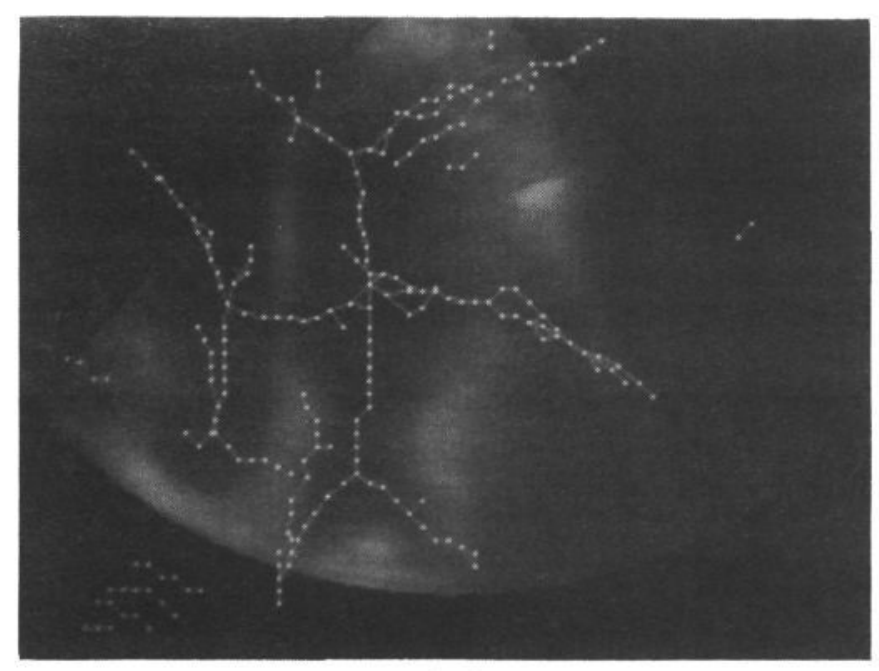

( a )

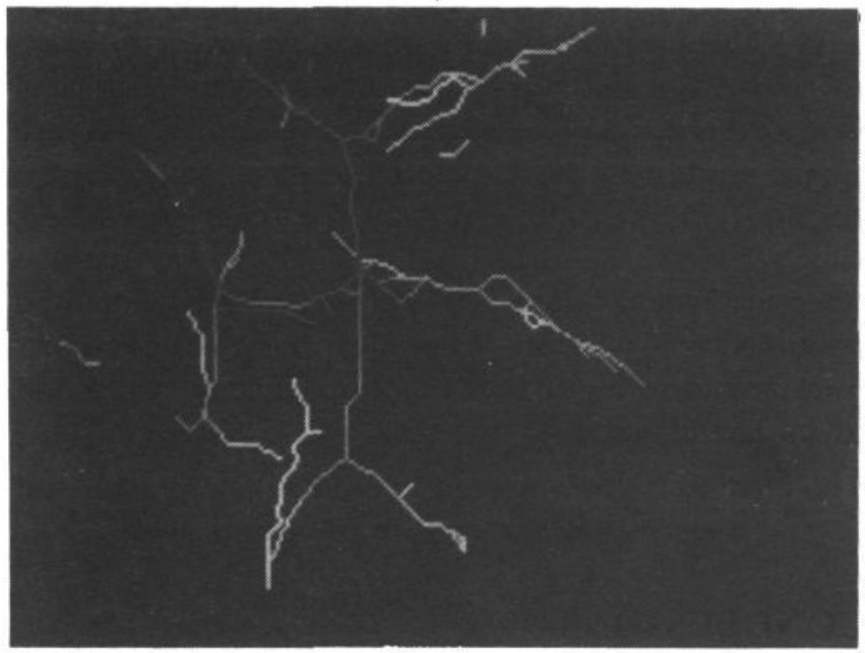

(c)

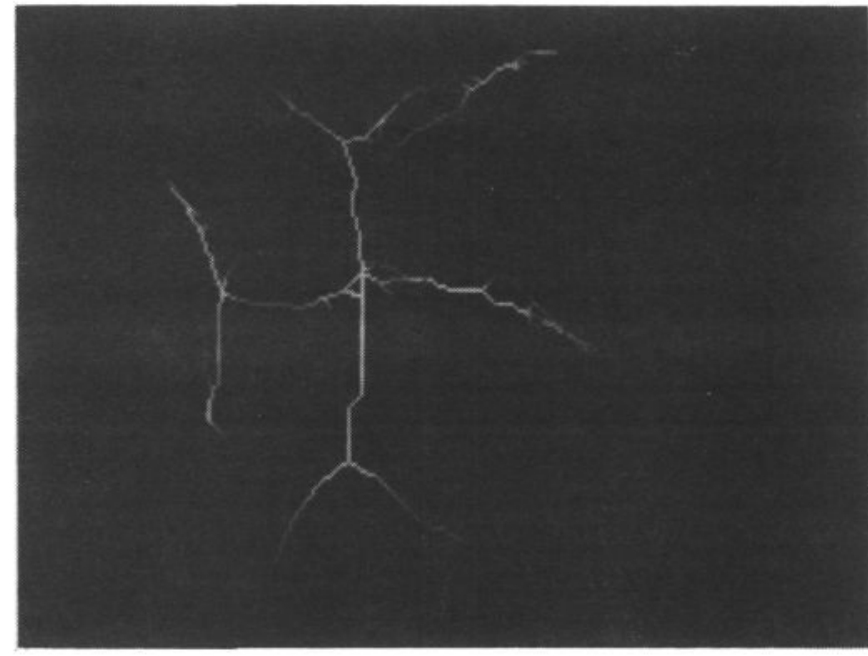

( b )

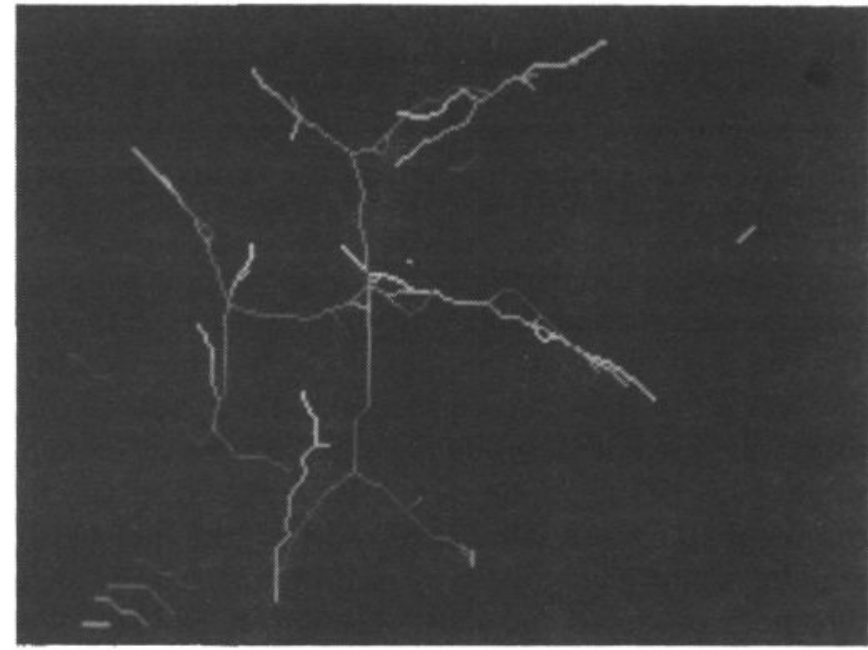

( d )

Figure 8. The grey level SAT of Fig 1a. (a) The gsat graph superimposed on the original image. The vertices are shown as bright dots, and the position of each vertex is the average spatial position of the associated surface path. (b), (c) and (d) show the other parameters which are associated with each vertex and describe the surface paths: (b) the difference in grey level between the top and the bottom of the path, (c) the grey level of the bottom of the path, (d) the average radius of the maximal discs.

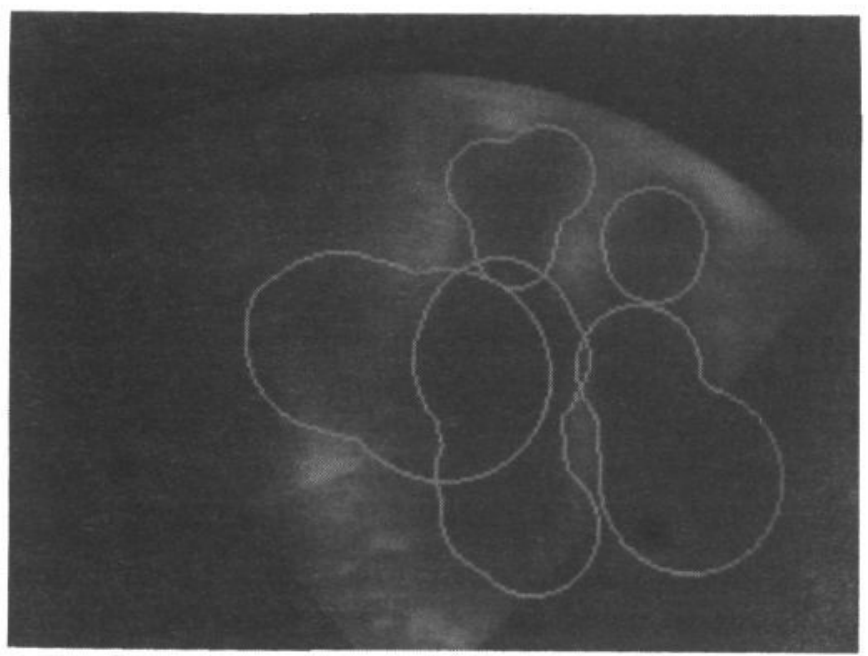

Figure 9. The boundaries of the 5 most salient cavities which were found using the grey level SAT
4. Gauch, J.M., and Pizer, S.M. "Image description via the multiresolution intensity axis of symmetry", Proc. of 2nd ICCV 1988, pp 269-274.

5. Peleg, S., and Rosenfeld, A. “A min-max medial axis transformation", IEEE Trans. PAMI, vol. 3 ( 1981 ), pp 208-210.

6. Toet, A. "A hierarchical morphological image decomposition”, Pattern Recognition Letters, vol. 11 ( 1990 ), pp 267-274.

7. Bailes, D.R. “The morphological grey level SAT", internal Alvey MMI-134 report: Mobprim/MU/Rep3/900110 ( 1990 ).

8. Goetcherian, V. "From binary to grey tone image processing using fuzzy logic concepts”, Pattern Recognition, vol. 12 ( 1980 ), pp 7-15. 